\title{
BMJ Open Prevalence of tinnitus and/or hyperacusis in children and adolescents: study protocol for a systematic review
}

\author{
Susanne Steen Nemholt, ${ }^{1,2}$ Jesper Hvass Schmidt, ${ }^{1,2,3}$ Niels Wedderkopp, ${ }^{4,5}$ \\ David M Baguley ${ }^{6,7}$
}

To cite: Nemholt S,

Schmidt JH, Wedderkopp N, et al. Nemholt SS. Prevalence of tinnitus and/or hyperacusis in children and adolescents: study protocol for a systematic review. BMJ Open 2015;5:e006649.

doi:10.1136/bmjopen-2014006649

- Prepublication history and additional material is available. To view please visit the journal (http://dx.doi.org/ 10.1136/bmjopen-2014006649).

Received 16 September 2014 Revised 19 November 2014 Accepted 20 November 2014

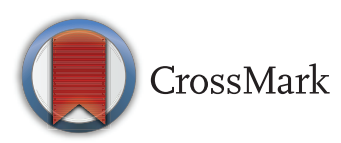

For numbered affiliations see end of article.

Correspondence to Susanne Steen Nemholt; snemholt@health.sdu.dk

\section{ABSTRACT}

Introduction: There is some debate as to what extent epidemiological data for the prevalence of childhood tinnitus can be relied on. While indications are that the prevalence is relatively high, referral numbers for children with tinnitus are reported to be low and many of the studies have a number of methodological difficulties. We describe the protocol of a systematic review aimed at assessing the prevalence of tinnitus and/or hyperacusis in children and young people.

Methods and analysis: We will include studies of any design (except case reports or case series) comparing the prevalence of tinnitus and/or hyperacusis in children and young people with and without hearing loss, any known external exposure and psychological disorders. We will search the following databases: PubMed, EMBASE and Scopus. No restrictions of language will be applied in the search strategy but during the article selection language is limited to English, German and Scandinavian languages. Primary and additional outcomes will be the prevalence of tinnitus/hyperacusis and the severity, respectively.

Ethics and dissemination: No ethical issues are foreseen. The results will be published in a peerreviewed journal and presented at national and international conferences of audiology and paediatrics. Trail registration number: This review protocol is registered in the PROSPERO International Prospective Register of Systematic Reviews, registration number CRD42014013456.

\section{INTRODUCTION}

Tinnitus is a symptom defined as the experience of perceiving sounds without any known audible external sound, ${ }^{1}$ which is the definition used in this article. Tinnitus can be perceived in one ear or both ears or inside the head. In order to eliminate cases with short duration ringing in the ear, the most commonly used question in epidemiological studies is some version of 'tinnitus lasting for more than $5 \mathrm{~min}$ at a time'. ${ }^{2}$ For the adult population the prevalence of tinnitus falls in

\section{Strengths and limitations of this study}

- Clearly established purpose, as well as a systematic and transparent approach.

- Comprehensive search strategy with search and data extraction conducted independently by two authors.

- During the article selection, language is limited to English, German and Scandinavian languages.

- This study will gather well-known published studies to determine the prevalence of tinnitus and/or hyperacusis across studies. This knowledge is important to know the extent of the problem.

- We expect some heterogeneity between studies, which makes it difficult to compare across studies.

the range $10-15 \%$. Unfortunately, there has been no consensus regarding definition of tinnitus used for phrasing appropriate questions and the populations studied vary widely. ${ }^{1}{ }^{3}$ Factors like age, hearing status and previous noise induction influences the prevalence outcome. An yet unpublished attempt to calculate a worldwide-pooled prevalence estimate of tinnitus in adults has not been possible due to the wide variability in tinnitus definitions among studies. ${ }^{2}$ The number of people with troublesome tinnitus is lower than the overall prevalence, ${ }^{1}$ as many people have tinnitus that is not bothersome. It has been noted that the association between tinnitus annoyance and perceived intensity is weak. ${ }^{3}$

The prevalence of tinnitus in children has been reported and estimates range from $3 \%$ to $58 \%$. The available studies have a wide variability in the population studied, some reports considering children with hearing loss, psychological conditions or children who have been noise exposed in comparison with children where these conditions are not known. ${ }^{4}$

Hyperacusis is often present in association with tinnitus. Several definitions of the term hyperacusis are in general use. Jastrebroff and Hazel define hyperacusis as an abnormal sound sensitivity arising from within the 
auditory system, either peripheral or central. ${ }^{5}$ They suggest that decreased sound tolerance consists not only of hyperacusis, but it also consists of a fear of sound (which they refer to as phonophobia) or a strong dislike of sound (which they call misophonia). In their description, patients with misophonia or phonophobia will have abnormally strong reactions of the limbic and autonomic nervous system without involvement of the auditory system, as defined in hyperacusis. The term phonophobia is also used within the neurological literature to describe intolerance to migraine headaches, adding to further confusion in its own real definition. ${ }^{6}$ Jastrebroff and Hazel use the factor of fear to disambiguate hyperacusis from phonophobia, which can be troublesome in the sense that it lacks the possible influence of context. Environmental factors include both the perceived content of sound (eg, sound intensity and quality) and support and relationships. Another attempt to define hyperacusis is from Coelho et $a l^{7}$ defining hyperacusis as lowered loudness discomfort levels associated with an abnormal annoyance to sounds. Baguley has described hyperacusis as an abnormal, lowered tolerance to sound, ${ }^{8}$ a definition which others have supported. ${ }^{9}$ This definition gives the possibility of including factors like mood and context to understand the variation of decreased sound tolerances between and within individuals, and will be used in this article.

The prevalence studies in this area are, given the complexity and inadequacy of terminology and definitions, diverse and frugal. The scarce statistics show an approximate prevalence of about $10-15 \% .{ }^{10}$ Approximately $40 \%$ of people with tinnitus also experience hyperacusis. ${ }^{11}$ The reported range of co-incidence of hyperacusis and tinnitus is very variable and can vary from $7.3 \%$ to $79 \% .^{12}$

Prevalence studies of childhood hyperacusis are even sparser. Coelho et $a l^{7}$ found a prevalence of $3.2 \%$ using their previous introduced defection on a population of 506 children, age 5-12 years. An unpublished study on 7.09311 years old children living in Bristol finds the prevalence of hyperacusis to be $3.68 \%$ using the question: "Do you ever experience oversensitivity or distress to particular sounds?" (D Baguley, personal communication, 2014).

There is some debate as to what extent epidemiological data for the prevalence of childhood tinnitus and hyperacusis can be relied on. Although some studies indicate a high prevalence, there is a low rate of spontaneous complaints and a recent study shows that the number of children seen with a primary complaint of tinnitus represents just a small fraction of the total number of patients seen for tinnitus in four European clinics with an established and internationally known tinnitus programme. ${ }^{13}$

This calls for caution when the epidemiological data for the prevalence is interpreted and indicates that it is important to attempt to understand what underpins the variation. Accurate estimates of the true prevalence are of value in planning diagnostic and intervention services.

It has been stated that the prevalence figures vary widely depending on the populations studied, methodologies used and the definition of tinnitus. The majority of the studies have not ascertained tinnitus severity and/ or complaint behaviour. The age range of children studied varies and there is a big degree of difference between definitions (tinnitus, hyperacusis) and measures (severity, perception, annoyance). It has been suggested that difficulties in interviewing children may lead to different answers. ${ }^{14}$ It is possible that study design, the definition of tinnitus and differences related to the included participants explain the inconsistencies in currently available studies.

\section{OBJECTIVES}

The aims are to assess the degree of variation among prevalence studies of tinnitus and hyperacusis in children, and to provide an overall summary of prevalence diversity.

We will conduct a systematic review of published studies to address the following objectives:

- To systematically review studies of the epidemiology of tinnitus and hyperacusis in children and young people in order to establish the reported prevalence estimates;

- To determine factors implicated in the variability of estimates, including those deriving from definitions;

- To investigate which methodological factors may determine differences in prevalence estimates.

We want to find possible explanations for the high degree of variation between different epidemiological studies to address the main study question: 'Is the prevalence of tinnitus and/or hyperacusis in children/young people (aged 5-19 years) higher in individuals, who have either hearing loss, psychological conditions or have been noise exposed in relation to children with tinnitus and/or hyperacusis where these conditions are not known?' Since the variable 'severity' is generally less reported than 'perception', we will explore the prevalence of chronic bothersome tinnitus whenever possible.

\section{METHODS}

Methods for this systematic review have been developed according to recommendations from the PRISMA checklists and the PRISMA Flow Diagram will be used to describe the flow of information through the different phases of the systematic review. ${ }^{15}$ This paper uses systematic and quantitative methods to examine reasons for variation in prevalence estimates.

\section{Selection criteria}

Study type

Studies will be selected and screened according to the research question and PICOS criteria. They will be included if they are original articles from peer-reviewed scientific journals published in English, German, Swedish, Norwegian or Danish.

\section{Population}

Children and young people aged 5-19 years will be included. If the age included in the studies falls outside 
this range, the study will be included if the prevalence is rated for different ages.

Comparisons: All studies, excluding case series and case studies, will be considered.

\section{Outcome}

The primary outcome will be the point prevalence of tinnitus and/or hyperacusis in individuals aged 5-19 years. The secondary outcome will be the consequences of tinnitus and/or hyperacusis (severity, annoyance, bothersome).

\section{Exclusion criteria}

Case study: children under the age of 5 and over the age of 19 years.

\section{Search methods for identification of studies Electronic searches}

The strategy for the electronic search has been developed with the assistance of librarians from the University of Southern Denmark. The first author will perform electronic searches in the databases PubMed, EMBASE and SCOPUS. No language period of publication limitations will be applied in the initial search.

The search period will be from 1960 to 2014. Our search has no restriction in the search period, as we want to include all studies in the review.

\section{PubMed search}

The matrix includes the relevant diagnoses (first category) and the topic of the studies we want to review (second category). As PubMed uses specific terms for various age groups, filters will be used to get the right population. Table 1 lists the search words used in the PubMed search.

The following MeSH terms and/or free text syntax will be used for PubMed

(("hyperacusis"[MeSH Terms] OR "hyperacusis"[All Fields]) OR ("tinnitus" [MeSH Terms] OR "tinnitus"[All
Fields]) OR misophonia[All Fields] OR ("hyperacusis" [MeSH Terms] OR "hyperacusis"[All Fields]) OR "phonophobia"[All Fields])) AND ((“epidemiology" [Subheading] OR "epidemiology"[All Fields] OR "epidemiology" [MeSH Terms]) OR ("epidemiology"

[MeSH Terms] OR "epidemiology"[All Fields] OR "epidemiologic"[All Fields]) OR ("epidemiology" [Subheading] OR "epidemiology"[All Fields] OR "prevalence"[All Fields] OR "prevalence"[MeSH Terms]) OR ("epidemiology" [Subheading] OR "epidemiology"[All Fields] OR "morbidity" [All Fields] OR "morbidity" [MeSH Terms]) OR ("epidemiology"[Subheading] OR "epidemiology"[All Fields] OR "occurrence"[All Fields] OR “epidemiology"[MeSH Terms] OR "occurrence"[All Fields]) OR ("epidemiology”[Subheading] OR "epidemiology"[All Fields] OR "incidence"[All Fields] OR "incidence" [MeSH Terms])) AND (("infant" [MeSH Terms] OR "child"[MeSH Terms] OR "children" [All Fields] OR "adolescent" [MeSH Terms]) OR "adolescence"[All Fields] OR "young adult"[MeSH Terms])

\section{EMBASE and SCOPUS search}

The matrix includes the relevant diagnoses (first category), the topic of the studies we want to review (second category), and the relevant population groups (third category). Table 2 lists the search words used for EMBASE and SCOPUS search.

The search terms and syntax for EMBASE and SCOPUS will be as follow:

(Tinnitus or Hyperacus* OR misophonia OR phonophobia) AND (epidemiology OR epidemiologic OR prevalence or morbidity OR occurrence OR incidence) AND (infant OR child OR children OR adolescent OR adolescence OR young adult)

\section{Searching other resources}

Manual searches will include scanning of reference lists of relevant papers.

Table 1 Matrix for PubMed search

\begin{tabular}{|c|c|c|c|c|c|}
\hline $\begin{array}{l}\text { First category } \\
\text { Tinnitus/hyperacusis }\end{array}$ & $\mathrm{N}=$ & & $\begin{array}{l}\text { Second category } \\
\text { Epidemiology }\end{array}$ & $\mathrm{N}=$ & Filter* \\
\hline $\begin{array}{l}\text { Tinnitus } \\
\text { OR } \\
\text { Hyperacusis } \\
\text { OR } \\
\text { Hyperacousis } \\
\text { OR } \\
\text { Misophonia } \\
\text { OR } \\
\text { Phonophobia }\end{array}$ & $\begin{array}{l}(\mathrm{n}=) \\
(\mathrm{n}=) \\
(\mathrm{n}=) \\
(\mathrm{n}=)\end{array}$ & AND & $\begin{array}{l}\text { Epidemiology } \\
\text { OR } \\
\text { Prevalence } \\
\text { OR } \\
\text { Morbidity } \\
\text { OR } \\
\text { Occurrence } \\
\text { OR } \\
\text { Incidence }\end{array}$ & $\begin{array}{l}(\mathrm{n}=) \\
(\mathrm{n}=) \\
(\mathrm{n}=) \\
(\mathrm{n}=) \\
(\mathrm{n}=)\end{array}$ & $\begin{array}{l}\text { Preschool child } \\
\text { Child } \\
\text { Adolescents } \\
\text { Young Adults }\end{array}$ \\
\hline
\end{tabular}


Table 2 Matrix for EMBASE and SCOPUS search

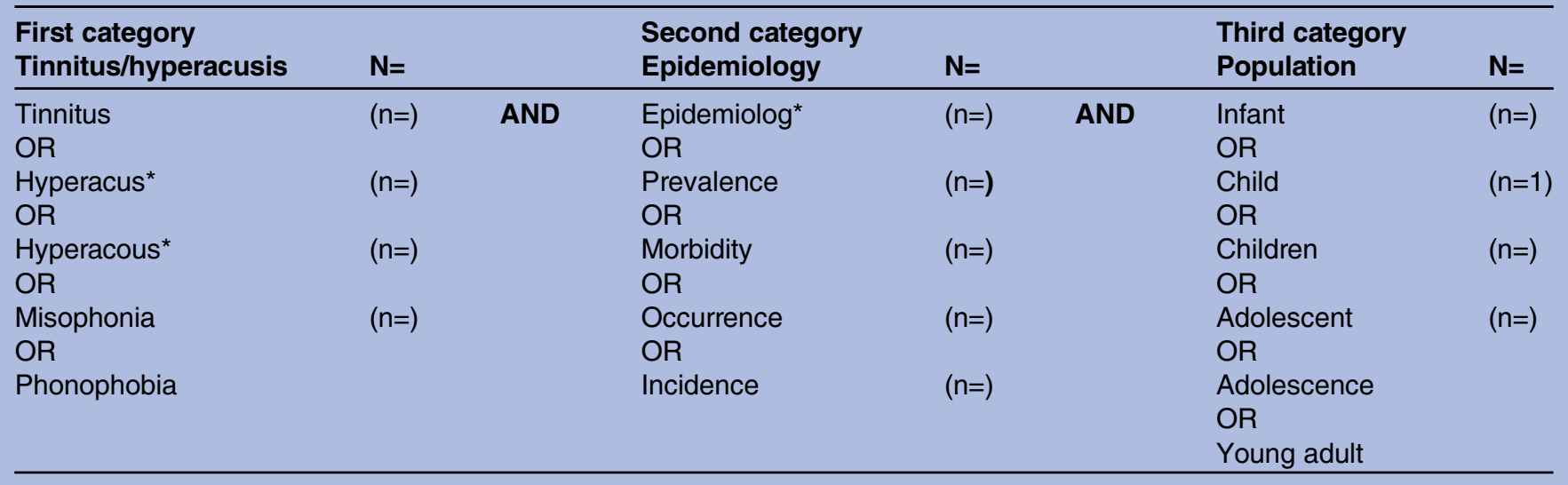

\section{Identification and selection of studies}

Studies identified with electronic and manual searches will be listed with citation, titles and abstracts in Endnote. Duplicates will be found using the Endnote function 'find duplicates' to compare each set of duplicate references. Figure 1 provides an overview of the search flow chart.

The eligibility process will be conducted in two separate stages.

Figure 1 Search flow chart (PRISMA flow diagram).
1. Two authors (SN and JS) will independently screen title and abstracts of all non-duplicated papers and will exclude those not pertinent. A final list will be agreed with discrepancies resolved by consensus between the two authors. When consensus is not reached, a third author (DB or NW) will act as arbitrator. If any doubt about inclusion exists, the article will proceed to the next stage.

\section{Records identified through database searching} $(n=)$
Additional records identified through other sources $(n=)$
Records after duplicates removed $(n=)$

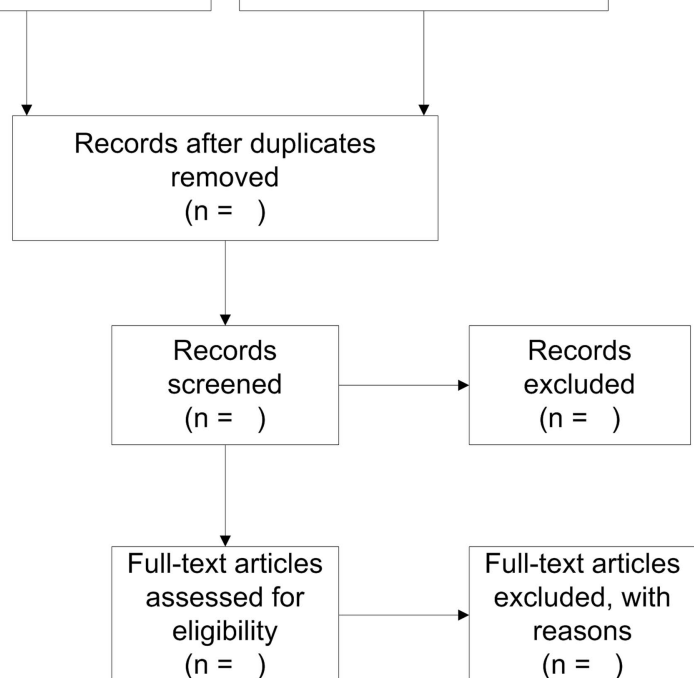

Studies included in qualitative synthesis $(n=)$ 
2. Agreement between the two authors will be assessed and reported using $\kappa$-statistics and overall agreement.

3. The full-text version of the articles passing stage 1 screening will be downloaded and assessed for eligibility by two authors ( $\mathrm{SN}$ and JS), independently. Discrepancies will be resolved by consensus between the two authors and if needed, a third author (DB or NW) will act as arbitrator.

\section{DATA EXTRACTION}

Two researchers (SN and JS) will independently perform data extraction; any discrepancies will be resolved by consensus between the two authors. If this is not possible, another author (DB or NW) will make a judgement on the data entered and act as an arbitrator.

\section{Definition of checklist items}

The principles from Grading of Recommendations Assessment, Development and Evaluation (GRADE) are useful in order to evaluate the overall quality of evidence

\section{Box 1 Descriptive items}

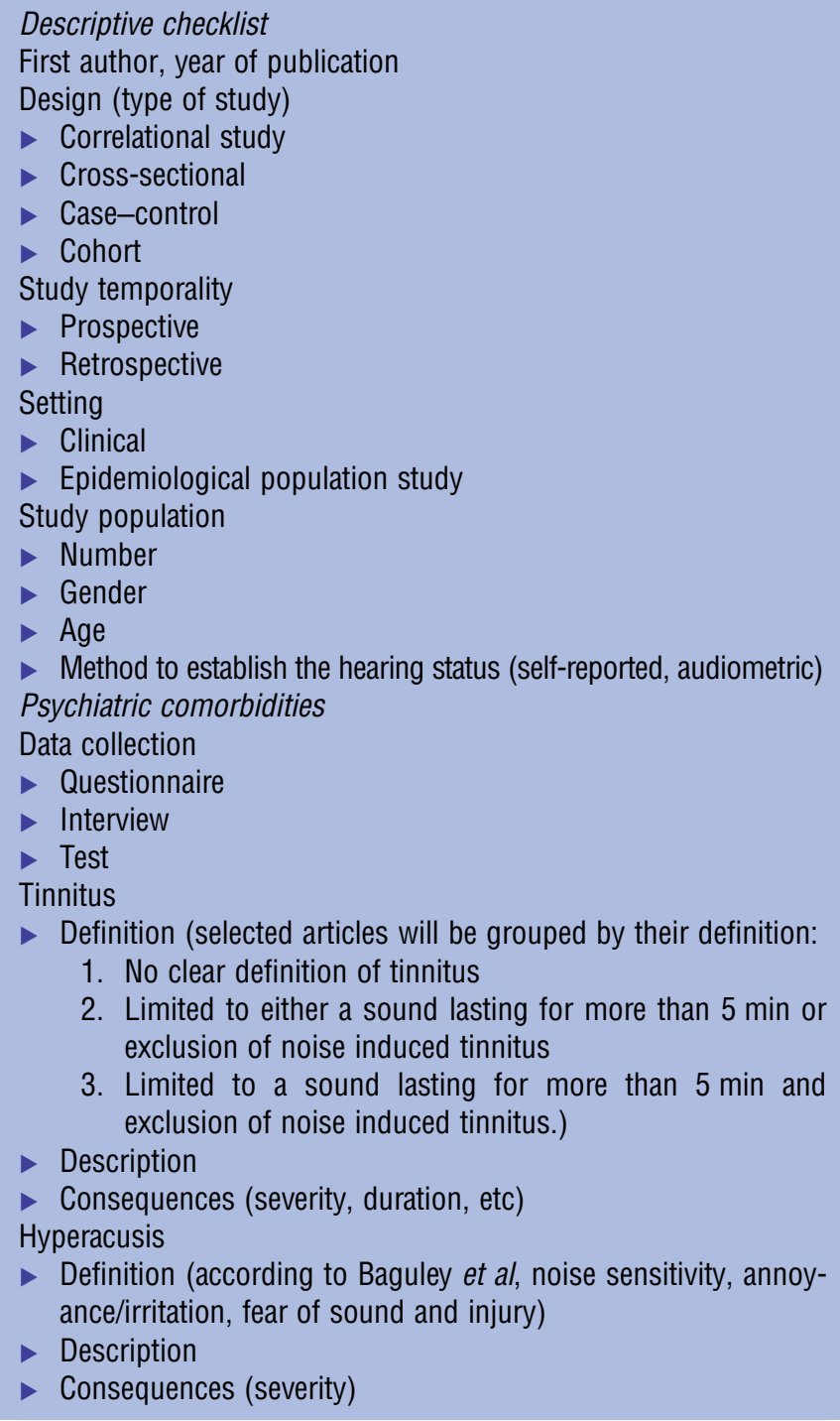

Table 3 Quality items

Quality checklist

Yes No

Clear tinnitus/hyperacusis definition

Tinnitus/hyperacusis well defined to

participants

Tested for question understood by child

Clear separation between tinnitus/hyperacusis

perception and tinnitus/hyperacusis severity

Clear separation of aspects of tinnitus

for the studies ${ }^{16}$ and will be used if possible. It is most likely, however, that the quality of the studies is unsuited to evaluate using GRADE. In that case, relevant articles will be reviewed according to the following checklist, in which the authors have defined a set of criteria consisting of descriptors, quality items and study results that is considered essential for this review. Box 1 shows the descriptive items that will be extracted. Table 3 provides an overview of the quality items and table 4 shows the results checklist. Assessment of study quality and bias in included studies is shown in online supplementary file 1.

Data will be extracted and inserted in an Excel sheet.

1. Descriptive items

2. Quality items

No fixed set of generally accepted quality criteria has been found that suits this type of literature review and therefore quality criteria has been chosen in consideration of factors important for a systematic review of prevalence. We want to look at the following:

- Which definition of tinnitus/hyperacusis is used and how is it diagnosed?

- Does the study provide a clear separation between tinnitus/hyperacusis perception and severity?

- Another possible reason for the prevalent differences could be found in the variations of studied groups. Therefore, we will compare the prevalence findings of the studies in relation to the selected participants: normal hearing, hearing loss, noise exposure and psychological problems.

3 Results

\begin{tabular}{l} 
Table 4 Results \\
\hline Prevalence $\quad$ Tinnitus Annoyance Hyperacusis \\
\hline All \\
Boys \\
Girls \\
$<13$ years \\
$>13$ years \\
Normal hearing \\
Hearing impaired \\
Noise induced \\
Data collection \\
Questionnaire \\
Interview \\
Preformed \\
hearing test
\end{tabular}


Author affiliations

${ }^{1}$ Institute of Clinical Research, University of Southern Denmark, and The Social Enterprise, The Capital Region of Denmark, Copenhagen, Denmark ${ }^{2}$ Department of Audiology, Odense University Hospital, Odense, Denmark ${ }^{3}$ Department of ENT Head and Neck Surgery, Odense University Hospital, Odense, Denmark

${ }^{4}$ Orthopedic Department, Sport Medicine Clinic, Hospital of Lillebaelt Institute of Regional Health Service Research, Odense, Denmark

${ }^{5}$ Center for Research in Childhood Health University of Southern Denmark, Odense, Denmark

${ }^{6}$ Department of Audiology, Cambridge University Hospitals, Cambridge, UK ${ }^{7}$ Department of Hearing and Vision Sciences, Anglia Ruskin University, Cambridge, UK

Contributors SSN, JHS were involved in study design, implementation, analysis and writing. NW and DMB were involved in study design, writing and editing.

Funding This review is part of a doctoral study which has been funded by The Capital Region of Denmark, The University of Southern Denmark and The Danish Association of the Hard of Hearing.

Competing interests None.

Provenance and peer review Not commissioned; externally peer reviewed.

Data sharing statement All recorded data from the data extraction process will be available on request to the extent that it is not included in the systematic review article.

Open Access This is an Open Access article distributed in accordance with the Creative Commons Attribution Non Commercial (CC BY-NC 4.0) license, which permits others to distribute, remix, adapt, build upon this work noncommercially, and license their derivative works on different terms, provided the original work is properly cited and the use is non-commercial. See: http:// creativecommons.org/licenses/by-nc/4.0/

\section{REFERENCES}

1. Henry JA, Dennis KC, Schechter MA. General review of tinnitus: prevalence, mechanisms, effects, and management. J Speech Lang Hear Res 2005;48:1204-35.

2. McCormack A, Edmondson-Jones M, Wadsworth S, et al. A systematic review of the prevalence of tinnitus. Poster Presentation at the IX International Tinnitus Seminar, Berlin, 2014.

3. Baguley D, McFerran D, Hall D. Tinnitus. Lancet 2013;382:1600-7.

4. Coelho CB. Epidemiology of tinnitus in children. Chapter 6 , In: Moller AR, ed. Textbook of Tinnitus. Springer, 2010:39-44.

5. Jastreboff PJ, Hazell JW. A neurophysiological approach to tinnitus: clinical implications. Br J Audiol 1993;27:7-17.

6. Asha'ari ZA, Mat Zain N, Razali A. Phonophobia and hyperacusis: practical points from a case report. Malays J Med Sci 2010;17:49-51.

7. Coelho CB, Sanchez TG, Tyler RS. Hyperacusis, sound annoyance, and loudness hypersensitivity in children. Prog Brain Res 2007:166:169-78

8. Baguley DM. Hyperacusis. J R Soc Med 2003;96:582-5.

9. Moller AR. Tinnitus: presence and future. Prog Brain Res 2007;166:3-16.

10. Knipper M, Van Dijk P, Nunes I, et al. Advances in the neurobiology of hearing disorders: recent developments regarding the basis of tinnitus and hyperacusis. Prog Neurobiol 2013;111:17-33.

11. Baguley D. Hyperacusis: an overview. Semin Hear 2014;35:74-83.

12. Guimaraes AC, Carvalho GM, Voltolini MM, et al. Study of the relationship between the degree of tinnitus annoyance and the presence of hyperacusis. Braz J Otorhinolaryngol 2014;80:24-8.

13. Baguley DM, Bartnik G, Kleinjung T, et al. Troublesome tinnitus in childhood and adolescence: data from expert centres. Int $J$ Pediatr Otorhinolaryngol 2013;77:248-51.

14. Stouffer JL, Tyler RS, Booth JC, et al. Tinnitus in normal-hearing and hearing-impaired children. Tinnitus 91: Proceedings of the Fourth International Tinnitus Seminar, 1992.

15. Liberati A, Altman DG, Tetzlaff J, et al. The PRISMA statement for reporting systematic reviews and meta-analyses of studies that evaluate healthcare interventions: explanation and elaboration. BMJ 2009;339:b2700.

16. Atkins D, Best D, Briss PA, et al. Grading quality of evidence and strength of recommendations. BMJ 2004;328:1490. 